\title{
Factorization theory for Wiener-Hopf plus Hankel operators with almost periodic symbols
}

\author{
A. P. Nolasco and L. P. Castro
}

\begin{abstract}
A factorization theory is proposed for Wiener-Hopf plus Hankel operators with almost periodic Fourier symbols. We introduce a factorization concept for the almost periodic Fourier symbols such that the properties of the factors will allow corresponding operator factorizations. Conditions for left, right, or both-sided invertibility of the Wiener-Hopf plus Hankel operators are therefore obtained upon certain indices of the factorizations. Under such conditions, the one-sided and two-sided inverses of the operators in study are also obtained.
\end{abstract}

\section{Historical background}

We would like to begin with a historical review about Hankel operators, including Toeplitz operators and Wiener-Hopf operators, till Wiener-Hopf plus Hankel operators. The story of Hankel operators starts with Hermann Hankel and with his Ph.D. thesis 13 published in 1861. Here Hankel studied determinants of infinite complex matrices with entries defined by $a_{j k}=a_{j+k}(j, k \geq 0)$, where $a=\left\{a_{j}\right\}_{j \geq 0}$ is a sequence of complex numbers. These matrices are the therefore called Hankel matrices and have the special form

$$
H_{M}=\left(\begin{array}{ccccc}
a_{0} & a_{1} & a_{2} & a_{3} & \ldots \\
a_{1} & a_{2} & a_{3} & a_{4} & \cdots \\
a_{2} & a_{3} & a_{4} & a_{5} & \cdots \\
a_{3} & a_{4} & a_{5} & a_{6} & \cdots \\
\vdots & \vdots & \vdots & \vdots & \ddots
\end{array}\right) .
$$

In 1881, Kronecker [14 presented a theorem that describes the Hankel matrices of finite rank as the ones that have corresponding power series,

$$
a(z)=\sum_{j=0}^{\infty} a_{j} z^{j}
$$

2000 Mathematics Subject Classification. 47B35, 47A68, 47A53, 42A75.

Key words and phrases. Wiener-Hopf plus Hankel operator, invertibility, factorization, almost periodic function.

A. P. Nolasco is sponsored by Fundação para a Ciência e a Tecnologia (Portugal) under grant number SFRH/BD/11090/2002. 
which are rational functions. The most famous Hankel matrix is the Hilbert matrix given by $\left\{\frac{1}{j+k+1}\right\}_{j, k \geq 0}$, i.e.,

$$
\mathcal{H}_{M}=\left(\begin{array}{ccccc}
1 & \frac{1}{2} & \frac{1}{3} & \frac{1}{4} & \cdots \\
\frac{1}{2} & \frac{1}{3} & \frac{1}{4} & & \ddots \\
\frac{1}{3} & \frac{1}{4} & & \ddots & \\
\frac{1}{4} & & \ddots & & \\
\vdots & \ddots & & &
\end{array}\right) .
$$

In 1906, Hilbert proved that the operator induced by the Hilbert matrix,

$$
\mathcal{H}: \ell^{2} \rightarrow \ell^{2},\left\{b_{j}\right\}_{j \geq 0} \mapsto\left\{\sum_{k=0}^{\infty} \frac{1}{j+k+1} b_{k}\right\}_{j \geq 0},
$$

is bounded on $\ell^{2}$. In this way, we may find here the origin of Hankel operators, arising from Hankel matrices. Considering $\left\{a_{j}\right\}_{j \geq 0} \in \ell^{2}$, the Hankel operator induced by the Hankel matrix $\left\{a_{j+k}\right\}_{j, k \geq 0}$ is defined by

$$
H: \ell^{2} \rightarrow \ell^{2},\left\{c_{j}\right\}_{j \geq 0} \mapsto\left\{\sum_{k=0}^{\infty} a_{j+k} c_{k}\right\}_{j \geq 0} .
$$

Later on, in 1957, Nehari presented a characterization for bounded Hankel operators on $\ell^{2}$ [17. Due to the importance of such characterization, we may say that it marks the beginning of the contemporary period of the study of Hankel operators. From Nehari's result, it follows the representation of two kinds of Hankel operators on the Hardy space on the unit circle, $H^{2}(\mathbb{T})$. For $\phi \in L^{2}(\mathbb{T})$, the Hankel operator $\Gamma_{\phi}: H^{2}(\mathbb{T}) \rightarrow H_{-}^{2}(\mathbb{T})$ is defined as

$$
\Gamma_{\phi}=\mathcal{P}_{-} \phi,
$$

with $H_{-}^{2}(\mathbb{T})=\left\{\varphi \in L^{2}(\mathbb{T}): \varphi_{n}=0, n>0\right\}$, where $\varphi_{n}=\frac{1}{2 \pi} \int_{0}^{2 \pi} \varphi\left(e^{i \theta}\right) e^{-i n \theta} d \theta$ is the $n$th Fourier coefficient of $\varphi \cdot \mathcal{P}_{-}$is the ortogonal projection from $L^{2}(\mathbb{T})$ onto $H_{-}^{2}(\mathbb{T})$,

$$
\mathcal{P}_{-}: \sum_{n=-\infty}^{\infty} \varphi_{n} t^{n} \mapsto \sum_{n=-\infty}^{-1} \varphi_{n} t^{n} .
$$

Considering the standard orthonormal basis for $H^{2}(\mathbb{T})$ and $H_{-}^{2}(\mathbb{T}), \Gamma_{\phi}$ has Hankel matrix $\left\{\varphi_{-j-k}\right\}_{j \geq 1, k \geq 0}$. For defining the other kind of Hankel operator, consider also $\phi \in L^{2}(\mathbb{T})$. The second Hankel operator $H_{\phi}: H^{2}(\mathbb{T}) \rightarrow H_{+}^{2}(\mathbb{T})$ is defined as

$$
H_{\phi}=\mathcal{P}_{+} \phi J_{\mathbb{T}},
$$

where $H_{+}^{2}(\mathbb{T})=\left\{\varphi \in L^{2}(\mathbb{T}): \varphi_{n}=0, n<0\right\}, \mathcal{P}_{+}$is the Riez projection from $L^{2}(\mathbb{T})$ onto $H_{+}^{2}(\mathbb{T})$,

$$
\mathcal{P}_{+}: \sum_{n=-\infty}^{\infty} \varphi_{n} t^{n} \mapsto \sum_{n=0}^{\infty} \varphi_{n} t^{n}
$$

and $J_{\mathbb{T}}$ is the flip operator in $L^{2}(\mathbb{T})$ given by $J_{\mathbb{T}} f(t)=t^{-1} f\left(t^{-1}\right), t \in \mathbb{T}$. In this case, the Hankel matrix of $H_{\phi}$ is the matrix $\left\{\varphi_{j+k}\right\}_{j, k \geq 0}$. 
Let us now go back in history to 1911. In this year, Otto Toeplitz published the paper [26] where he studied infinite linear systems of the form

$$
\left(\begin{array}{ccc|cccc}
\ddots & \vdots & \vdots & \vdots & \vdots & \vdots & . \cdot \\
\cdots & a_{0} & a_{-1} & a_{-2} & a_{-3} & a_{-4} & \cdots \\
\cdots & a_{1} & a_{0} & a_{-1} & a_{-2} & a_{-3} & \cdots \\
\hline \cdots & a_{2} & a_{1} & a_{0} & a_{-1} & a_{-2} & \cdots \\
\cdots & a_{3} & a_{2} & a_{1} & a_{0} & a_{-1} & \cdots \\
\cdots & a_{4} & a_{3} & a_{2} & a_{1} & a_{0} & \cdots \\
. & \vdots & \vdots & \vdots & \vdots & \vdots & \ddots
\end{array}\right)\left(\begin{array}{c}
\vdots \\
f_{-2} \\
f_{-1} \\
\hline f_{0} \\
f_{1} \\
f_{2} \\
\vdots
\end{array}\right)=\left(\begin{array}{c}
\vdots \\
g_{-2} \\
g_{-1} \\
\hline g_{0} \\
g_{1} \\
g_{2} \\
\vdots
\end{array}\right)
$$

on the space $\ell^{2}(\mathbb{Z})$. In this context, Toeplitz was interested in the study of the spectrum of the operator on $\ell^{2}(\mathbb{Z})$ generated by matrices of the form

$$
\left(\begin{array}{c|c}
B_{11} & B_{12} \\
\hline B_{21} & B_{22}
\end{array}\right)=\left(\begin{array}{ccc|cccc}
\ddots & \vdots & \vdots & \vdots & \vdots & \vdots & . \cdot \\
\cdots & a_{0} & a_{-1} & a_{-2} & a_{-3} & a_{-4} & \cdots \\
\cdots & a_{1} & a_{0} & a_{-1} & a_{-2} & a_{-3} & \cdots \\
\hline \cdots & a_{2} & a_{1} & a_{0} & a_{-1} & a_{-2} & \cdots \\
\cdots & a_{3} & a_{2} & a_{1} & a_{0} & a_{-1} & \cdots \\
\cdots & a_{4} & a_{3} & a_{2} & a_{1} & a_{0} & \cdots \\
. & \vdots & \vdots & \vdots & \vdots & \vdots & \ddots
\end{array}\right),
$$

which he called "L-matrices", meaning Laurent matrices. Toeplitz matrices arise from the Laurent matrices when we consider in Laurent matrices the block $B_{22}$ :

$$
\mathcal{T}=\left(B_{22}\right)=\left(\begin{array}{cccc}
a_{0} & a_{-1} & a_{-2} & \cdots \\
a_{1} & a_{0} & a_{-1} & \cdots \\
a_{2} & a_{1} & a_{0} & \cdots \\
\vdots & \vdots & \vdots & \ddots
\end{array}\right) .
$$

As we can see, Toeplitz matrices are therefore defined by $a_{j k}=a_{j-k}$, where $a=$ $\left\{a_{j}\right\}_{j \in \mathbb{N}}$ is a sequence of complex numbers. Contrarily to what happens with Hankel matrices that are constant on every diagonal parallel to the secondary diagonal, Toeplitz matrices are constant on every diagonal parallel to the main diagonal. In the same way that Hankel operators were defined in $\ell^{2}$, Toeplitz operators on $\ell^{2}$ are defined similarly. Thus, the Toeplitz operator is defined as the operator induced on $\ell^{2}$ by the Toeplitz matrix (1.1). We may also define the Toeplitz operator on the Hardy space $H^{2}(\mathbb{T})$. In this sense, for $\phi \in L^{\infty}(\mathbb{T})$, the Toeplitz operator is defined as

$$
T_{\phi}=\mathcal{P}_{+} \phi: H^{2}(\mathbb{T}) \rightarrow H_{+}^{2}(\mathbb{T}) .
$$

Moving now to the thirties and to the integral operators, we find the work of Norbert Wiener and Eberhard Hopf. They presented in 1931 a method to solve integral equations of the form

$$
c f(x)+\int_{0}^{\infty} k(x-y) f(y) d y=g(x), \quad x \in \mathbb{R}_{+},
$$

i.e. the so-called Wiener-Hopf equations 27 . Here $c \in \mathbb{C}, k \in L^{1}(\mathbb{R})$ and $f, g \in$ $L^{2}\left(\mathbb{R}_{+}\right)$, where $c$ and $k$ are fixed, $g$ is given and $f$ is unknown. From Wiener-Hopf 
equations arise Wiener-Hopf operators defined by

$$
W_{\phi} f(x)=c f(x)+\int_{0}^{\infty} k(x-y) f(y) d y, \quad x \in \mathbb{R}_{+},
$$

where $\phi$ belongs to the Wiener algebra. The Wiener algebra is defined by

$$
\mathbb{W}=\left\{\phi: \phi=c+\mathcal{F} k, c \in \mathbb{C}, k \in L^{1}(\mathbb{R})\right\}
$$

and it is a Banach algebra under the norm $\|c+\mathcal{F} k\|_{\mathbb{W}}=|c|+\|k\|_{L^{1}(\mathbb{R})}$ and the usual multiplication operation. The Wiener-Hopf operators are convolution type operators. Therefore, they can also be represented as

$$
W_{\phi}=r_{+} \mathcal{F}^{-1} \phi \cdot \mathcal{F}: L_{+}^{2}(\mathbb{R}) \rightarrow L^{2}\left(\mathbb{R}_{+}\right) .
$$

Here $L_{+}^{2}(\mathbb{R})$ denotes the subspace of $L^{2}(\mathbb{R})$ formed by all the functions supported in the closure of $\mathbb{R}_{+}=(0,+\infty), r_{+}$is the restriction operator from $L_{+}^{2}(\mathbb{R})$ into $L^{2}\left(\mathbb{R}_{+}\right)$and $\mathcal{F}$ denotes the Fourier transformation. The Wiener-Hopf operators on $L_{+}^{2}(\mathbb{R})$ may also be seen in the form

$$
\begin{aligned}
W_{\phi} & =\ell_{0} r_{+} \mathcal{F}^{-1} \phi \cdot \mathcal{F}: L_{+}^{2}(\mathbb{R}) \rightarrow L_{+}^{2}(\mathbb{R}) \\
& =P_{+} A: L_{+}^{2}(\mathbb{R}) \rightarrow L_{+}^{2}(\mathbb{R})
\end{aligned}
$$

with $\ell_{0}: L^{2}\left(\mathbb{R}_{+}\right) \rightarrow L^{2}(\mathbb{R})$ being the zero extension operator, $P_{+}=\ell_{0} r_{+}$being the canonical projection of $L^{2}(\mathbb{R})$ onto $L_{+}^{2}(\mathbb{R})$ and where $A$ is the translation invariant operator $\mathcal{F}^{-1} \phi \cdot \mathcal{F}$. Looking now to (1.2) and (1.3), we see that Wiener-Hopf operators and Toeplitz operators may be interpreted in the same way.

An important step in the study of Toeplitz (or Wiener-Hopf) and Hankel operators was made in 1979 when Power 19 used the $C^{*}$-algebra generated by the Toeplitz and Hankel operators. In that paper Power devoted already a particular attention to those kind of operators when generated by piecewise continuous functions. Later on several authors considered also the interactions between Wiener-Hopf and Hankel operators, as well as the algebra generated by them, see e.g. 1, 2, 4, 20, 21, 22, 23.

In the decade of 90 , we find several papers related to diffraction problems where Wiener-Hopf plus Hankel operators appear as the associated operators of the systems of equations in $L^{2}(\mathbb{R})$ to which the diffraction problems can be reduced to (cf. 15, 16, 25]). Such concrete use of the Wiener-Hopf plus Hankel operators continues in our days and particular examples of their applicability in diffraction theory may be found in $\mathbf{7}, \mathbf{8}$.

As a consequence, the theory of Wiener-Hopf plus Hankel is nowadays well developed for some classes of Fourier symbols like the case of continuous or piecewise continuous functions. However, this is not the case for the almost periodic class. The present paper is an attempt to proceed with this last direction.

\section{The Main Objects}

The main objects of the present work are the Wiener-Hopf plus Hankel operators with Fourier symbols in the algebra of almost periodic functions, and acting between $L^{2}$ Lebesgue spaces. In a detailed way, we will consider operators with the form

$$
W H_{\phi}=W_{\phi}+H_{\phi}: L_{+}^{2}(\mathbb{R}) \rightarrow L^{2}\left(\mathbb{R}_{+}\right),
$$


with $W_{\phi}$ and $H_{\phi}$ being Wiener-Hopf and Hankel operators defined by

$$
\begin{aligned}
W_{\phi} & =r_{+} \mathcal{F}^{-1} \phi \cdot \mathcal{F}: L_{+}^{2}(\mathbb{R}) \rightarrow L^{2}\left(\mathbb{R}_{+}\right) \\
H_{\phi} & =r_{+} \mathcal{F}^{-1} \phi \cdot \mathcal{F} J: L_{+}^{2}(\mathbb{R}) \rightarrow L^{2}\left(\mathbb{R}_{+}\right),
\end{aligned}
$$

respectively. Here and in what follows, $J$ is the reflection operator given by the rule $J \varphi(x)=\widetilde{\varphi}(x)=\varphi(-x), x \in \mathbb{R}$.

The so-called Fourier symbol $\phi$ belongs to the algebra of the almost periodic functions $A P$, that is, the smallest closed subalgebra of $L^{\infty}(\mathbb{R})$ that contains all the functions $e_{\lambda}(\lambda \in \mathbb{R})$ where $e_{\lambda}(x)=e^{i \lambda x}, x \in \mathbb{R}$.

Let us determine the notation $\mathcal{G} B$ for the group of all invertible elements of a Banach algebra $B$. By Bohr's theorem, for each $\phi \in \mathcal{G A P}$ there exists a real number $\kappa(\phi)$ and a function $\psi \in A P$ such that

$$
\phi(x)=e^{i \kappa(\phi) x} e^{\psi(x)}, \quad x \in \mathbb{R} .
$$

Since $\kappa(\phi)$ is uniquely determined, $\kappa(\phi)$ is usually called the mean motion of $\phi$.

Let $A: X \rightarrow Y$ be a bounded linear operator acting between Banach spaces. If $\operatorname{Im} A$ is closed, the cokernel of $A$ is defined as Coker $A=Y / \operatorname{Im} A$. Then the operator $A$ is said to be properly d-normal if $\operatorname{dim} \operatorname{Coker} A$ is finite and $\operatorname{dim} \operatorname{Ker} A$ is infinite, properly $n$-normal if $\operatorname{dim} \operatorname{Ker} A$ is finite and $\operatorname{dim}$ Coker $A$ is infinite, and Fredholm if both $\operatorname{dim} \operatorname{Ker} A$ and $\operatorname{dim}$ Coker $A$ are finite.

For Wiener-Hopf operators with Fourier symbols in $\mathcal{G} A P$, there is a semiFredholm and invertibility criterion due to Gohberg-Feldman/Coburn-Douglas (cf. 10 and 12 or 3. Theorem 2.28]) based on the sign of the mean motion of the Fourier symbol of the operator. That criterion says that:

(1) if the mean motion of the Fourier symbol is negative, then the WienerHopf operator is properly $d$-normal and right-invertible;

(2) if the mean motion of the symbol is positive, then the Wiener-Hopf operator is properly $n$-normal and left-invertible;

(3) and in the case where the mean motion of the symbol is zero, then the Wiener-Hopf operator is invertible.

Such criterion was one of the starting motivations for the present work. Accordingly, the main purpose of this paper is to establish an invertibility criterion for WienerHopf plus Hankel operators with almost periodic Fourier symbols.

\section{Operator Identities for Wiener-Hopf plus Hankel Operators and their Invertibility in the Even Fourier Symbol Case}

In this section we will identify certain operator identities for Wiener-Hopf plus Hankel operators and Wiener-Hopf operators. Besides some particular consequences already obtained in the present section, this will also help us in the next sections in the process of obtaining an invertibility criterion for the Wiener-Hopf plus Hankel operators.

According to (2.1), (2.2) and (2.3), we have

$$
W H_{\phi}=r_{+}\left(\mathcal{F}^{-1} \phi \cdot \mathcal{F}+\mathcal{F}^{-1} \phi \cdot \mathcal{F} J\right)=r_{+} \mathcal{F}^{-1} \phi \cdot \mathcal{F}\left(I_{L_{+}^{2}(\mathbb{R})}+J\right),
$$

where $I_{L_{+}^{2}(\mathbb{R})}$ denotes the identity operator in $L_{+}^{2}(\mathbb{R})$. Furthermore, since

$$
I_{L_{+}^{2}(\mathbb{R})}+J=\ell^{e} r_{+}
$$


where $\ell^{e}: L^{2}\left(\mathbb{R}_{+}\right) \rightarrow L^{2}(\mathbb{R})$ denotes the even extension operator, we may write the Wiener-Hopf plus Hankel operator as

$$
W H_{\phi}=r_{+} \mathcal{F}^{-1} \phi \cdot \mathcal{F} \ell^{e} r_{+} .
$$

From the Wiener-Hopf and Hankel operator theory, the following relations are known:

$$
\begin{aligned}
& W_{\phi \varphi}=W_{\phi} \ell_{0} W_{\varphi}+H_{\phi} \ell_{0} H_{\tilde{\varphi}}, \\
& H_{\phi \varphi}=W_{\phi} \ell_{0} H_{\varphi}+H_{\phi} \ell_{0} W_{\widetilde{\varphi}},
\end{aligned}
$$

where $\ell_{0}: L^{2}\left(\mathbb{R}_{+}\right) \rightarrow L^{2}(\mathbb{R})$ is the zero extension operator. Additionally, from the last two identities, it follows that

$$
W H_{\phi \varphi}=W_{\phi} \ell_{0} W H_{\varphi}+H_{\phi} \ell_{0} W H_{\tilde{\varphi}}
$$

and

$$
W H_{\phi \varphi}=W H_{\phi} \ell_{0} W H_{\varphi}+H_{\phi} \ell_{0} W H_{\widetilde{\varphi}-\varphi} .
$$

Let $H^{\infty}\left(\mathbb{C}_{-}\right)$denote the set of all bounded and analytic functions in $\mathbb{C}_{-}=$ $\{z \in \mathbb{C}: \operatorname{Im} z<0\}$ and $H_{-}^{\infty}(\mathbb{R})$ be the set of all functions in $L^{\infty}(\mathbb{R})$ that are non-tangential limits of elements in $H^{\infty}\left(\mathbb{C}_{-}\right)$. Similarly we will use $H_{+}^{\infty}(\mathbb{R})$. Due to (3.2), if we consider $\phi \in H_{-}^{\infty}(\mathbb{R})$ or $\varphi$ being an even function, then we obtain a multiplicative relation

$$
W H_{\phi \varphi}=W H_{\phi} \ell_{0} W H_{\varphi} .
$$

Note that if the Fourier symbol of a Wiener-Hopf operator admits a factorization of the form $\varphi_{-} \psi \varphi_{+}$, where $\varphi_{ \pm} \in H_{ \pm}^{\infty}(\mathbb{R})$ and $\psi \in L^{\infty}(\mathbb{R})$, it is possible to apply to the Wiener-Hopf operator the multiplicative property

$$
W_{\varphi_{-} \psi \varphi_{+}}=W_{\varphi_{-}} \ell_{0} W_{\psi} \ell_{0} W_{\varphi_{+}}
$$

(see e.g. 3. Proposition 2.17]). With a convenient change, it is possible to construct for Wiener-Hopf plus Hankel operators a corresponding result as that one known for Wiener-Hopf operators. In the present case we may apply the multiplicative property on the left if the left factor belongs to $H_{-}^{\infty}(\mathbb{R})$ and on the right if the right factor is an even function, as it is asserted in the following proposition.

Proposition 3.1. If $\varphi \in H_{-}^{\infty}(\mathbb{R})$ and $\psi, \phi \in L^{\infty}(\mathbb{R})$ such that $\phi=\widetilde{\phi}$, then it holds

$W H_{\varphi \psi \phi}=W H_{\varphi} \ell_{0} W H_{\psi \phi}=W H_{\varphi} \ell_{0} W H_{\psi} \ell_{0} W H_{\phi}=W_{\varphi} \ell_{0} W H_{\psi} \ell_{0} W H_{\phi}$.

Proof. Since $\varphi \in H_{-}^{\infty}(\mathbb{R})$, we may apply the already presented multiplicative relation for Wiener-Hopf plus Hankel operators, see (3.3). Thus

$$
W H_{\varphi \psi \phi}=W H_{\varphi} \ell_{0} W H_{\psi \phi} \text {. }
$$

In addition, since $\phi=\widetilde{\phi}$, it also follows from (3.3) that

$$
W H_{\psi \phi}=W H_{\psi} \ell_{0} W H_{\phi}
$$

From (3.4) and (3.5), we have that

$$
W H_{\varphi \psi \phi}=W H_{\varphi} \ell_{0} W H_{\psi} \ell_{0} W H_{\phi} .
$$

Since $\varphi \in H_{-}^{\infty}(\mathbb{R})$, we have $H_{\varphi}=0$ due to the structure of the Hankel operators. Therefore $W H_{\varphi}=W_{\varphi}$ and it follows from (3.6) that $W_{\varphi \psi \phi}=W_{\varphi} \ell_{0} W H_{\psi} \ell_{0} W H_{\phi}$. 
Proposition 3.2. If $\phi_{e} \in \mathcal{G} L^{\infty}(\mathbb{R})$ and $\widetilde{\phi_{e}}=\phi_{e}$, then $W H_{\phi_{e}}$ is invertible and its inverse is the operator $\ell_{0} W H_{\phi_{e}^{-1}} \ell_{0}: L^{2}\left(\mathbb{R}_{+}\right) \rightarrow L_{+}^{2}(\mathbb{R})$.

Proof. On one hand, we have

$$
W H_{\phi_{e} \cdot \phi_{e}^{-1}} \ell_{0}=W H_{1} \ell_{0}=W_{1} \ell_{0}=I_{L^{2}\left(\mathbb{R}_{+}\right)},
$$

where $I_{L^{2}\left(\mathbb{R}_{+}\right)}$represents the identity operator in $L^{2}\left(\mathbb{R}_{+}\right)$. On the other hand, since $\phi_{e} \in \mathcal{G} L^{\infty}(\mathbb{R})$ and $\widetilde{\phi_{e}}=\phi_{e}$, then $\widetilde{\phi_{e}^{-1}}=\phi_{e}^{-1}$ and therefore we may apply the multiplicative relation for Wiener-Hopf plus Hankel operators. So we have

$$
W H_{\phi_{e} \cdot \phi_{e}^{-1}}=W H_{\phi_{e}} \ell_{0} W H_{\phi_{e}^{-1}} .
$$

Thus, combining (3.7) and (3.8), we get that

$$
W H_{\phi_{e}} \ell_{0} W H_{\phi_{e}^{-1}} \ell_{0}=I_{L^{2}\left(\mathbb{R}_{+}\right)} \text {. }
$$

In the same way, we obtain that

$$
\ell_{0} W H_{\phi_{e}^{-1}} \ell_{0} W H_{\phi_{e}}=I_{L_{+}^{2}(\mathbb{R})} .
$$

Therefore, (3.9)-(3.10) show that $W H_{\phi_{e}}$ is invertible and its inverse is $\ell_{0} W H_{\phi_{e}^{-1}} \ell_{0}$.

\section{AP Asymmetric Factorization}

Let $A P^{-}\left(A P^{+}\right)$denote the smallest closed subalgebra of $L^{\infty}(\mathbb{R})$ that contains all the functions $e_{\lambda}$ with $\lambda \leq 0(\lambda \geq 0)$.

The following definition was initially motivated by the role of the so-called $A P W$ factorization in the theory of Wiener-Hopf operators with Fourier symbols in the subclass of almost periodic elements that allow a representation in the form of an absolutely convergent series [3], and by the recent works about Toeplitz plus Hankel operators [1, 11] and convolution type operators with symmetry [8, 9]. Additionally, it extends a corresponding concept introduced in $\mathbf{1 8}$ for the subclass of almost periodic Fourier symbols that can be written as an absolutely convergent series.

Definition 4.1. We will say that a function $\phi \in \mathcal{G} A P$ admits an $A P$ asymmetric factorization if it can be represented in the form

$$
\phi=\phi_{-} e_{\lambda} \phi_{e}
$$

where $\lambda \in \mathbb{R}, e_{\lambda}(x)=e^{i \lambda x}, x \in \mathbb{R}, \phi_{-} \in \mathcal{G} A P^{-}, \phi_{e} \in \mathcal{G} L^{\infty}(\mathbb{R})$ with $\widetilde{\phi_{e}}=\phi_{e}$.

The particular case of an $A P$ asymmetric factorization with $\lambda=0$ will be referred to as a canonical AP asymmetric factorization.

It is interesting to clarify that, when existing, the $A P$ asymmetric factorization here introduced is unique up to a constant.

Proposition 4.2. Let $\phi \in \mathcal{G} A P$. Suppose that $\phi$ admits two $A P$ asymmetric factorizations:

$$
\begin{aligned}
& \phi=\phi_{-}^{(1)} e_{\lambda_{1}} \phi_{e}^{(1)}, \\
& \phi=\phi_{-}^{(2)} e_{\lambda_{2}} \phi_{e}^{(2)} .
\end{aligned}
$$

Then $\lambda_{1}=\lambda_{2}, \phi_{-}^{(1)}=\gamma \phi_{-}^{(2)}$ and $\phi_{e}^{(1)}=\gamma^{-1} \phi_{e}^{(2)}, \gamma \in \mathbb{C} \backslash\{0\}$. 
Proof. The equality $\phi_{-}^{(1)} e_{\lambda_{1}} \phi_{e}^{(1)}=\phi_{-}^{(2)} e_{\lambda_{2}} \phi_{e}^{(2)}$, implies that

$$
\left(\phi_{-}^{(2)}\right)^{-1} \phi_{-}^{(1)} e_{\lambda_{1}}=e_{\lambda_{2}} \phi_{e}^{(2)}\left(\phi_{e}^{(1)}\right)^{-1} \text {. }
$$

Assume, without loss of generality, that $\lambda_{1} \leq \lambda_{2}$. Then $\lambda=\lambda_{1}-\lambda_{2} \leq 0$. From (4.1) it follows that

$$
\left(\phi_{-}^{(2)}\right)^{-1} \phi_{-}^{(1)} e_{\lambda}=\phi_{e}^{(2)}\left(\phi_{e}^{(1)}\right)^{-1}
$$

Since the right-hand side of (4.2) is an even function, $\left(\phi_{-}^{(2)}\right)^{-1} \phi_{-}^{(1)} e_{\lambda}$ is also an even function. Put

$$
\varphi=\left(\phi_{-}^{(2)}\right)^{-1} \phi_{-}^{(1)} .
$$

Thus $\varphi(x) e_{\lambda}(x)=\widetilde{\varphi}(x) \widetilde{e_{\lambda}}(x)$, i.e. $\varphi(x) e_{\lambda}(x)=\widetilde{\varphi}(x) e_{-\lambda}(x)$, or equivalently

$$
\varphi(x) e_{2 \lambda}(x)=\widetilde{\varphi}(x) .
$$

On one hand, since $\varphi \in \mathcal{G} A P^{-}$, we may apply the well-known characterization of $\mathcal{G} A P^{-}$which assures the existence of a $\psi \in A P^{-}$such that $\varphi=e^{\psi}$ (cf. e.g. 3 Lemma 3.4]). On the other hand, because $\widetilde{\varphi} \in \mathcal{G} A P^{+}$, by a corresponding characterization of $\mathcal{G} A P^{+}$, there exists a $\eta \in A P^{+}$such that $\tilde{\varphi}=e^{\eta}$. From (4.4), it follows that

$$
e^{\psi(x)+i 2 \lambda x}=e^{\eta(x)},
$$

which implies that $\lambda=0$ and $\psi \in A P^{-} \cap A P^{+}$, i.e., $\lambda_{1}=\lambda_{2}$ and $\psi$ is a complex constant function. From (4.3), we get $\phi_{-}^{(1)}=\gamma \phi_{-}^{(2)}$ with $\gamma \in \mathbb{C} \backslash\{0\}$. By (4.2), we obtain $\phi_{e}^{(1)}=\gamma^{-1} \phi_{e}^{(2)}$.

\section{Invertibility Criterion and (Lateral) Inverses for Wiener-Hopf plus Hankel Operators}

Here we start with a known definition for bounded linear operators $T: X_{1} \rightarrow$ $X_{2}$ and $S: Y_{1} \rightarrow Y_{2}$, acting between Banach spaces. The operators $T$ and $S$ are said to be equivalent if there are two boundedly invertible linear operators, $E: Y_{2} \rightarrow X_{2}$ and $F: X_{1} \rightarrow Y_{1}$, such that

$$
T=E S F .
$$

It follows from (5.1) that if two operators are equivalent, then they belong to the same regularity class $\mathbf{5}, \mathbf{6}, \mathbf{2 4}$. More precisely, one of these operators is invertible, one-sided invertible, Fredholm, properly $n$-normal, properly $d$-normal, one-sided regularizable, generalized invertible or normally solvable, if and only if the other operator enjoys the same property. This important consequence of the equivalence operator relations will have a global preponderance in the proof of the following criterion.

TheOREM 5.1. Let $\phi \in \mathcal{G} A P$ admit an $A P$ asymmetric factorization $\phi=$ $\phi_{-} e_{\lambda} \phi_{e}$.

(a) If $\lambda<0$, then $W_{\phi}$ is properly d-normal and right-invertible.

(b) If $\lambda>0$, then $W H_{\phi}$ is properly n-normal and left-invertible.

(c) If $\lambda=0$, then $W H_{\phi}$ is invertible. 
Proof. In the case where $\lambda<0$, we have that $e_{\lambda} \in A P^{-}$. Since $A P^{-}=$ $A P \cap H_{-}^{\infty}(\mathbb{R})$, it holds that $e_{\lambda} \in H_{-}^{\infty}(\mathbb{R})$ and hence

$$
W H_{\phi}=W_{\phi_{-}} \ell_{0} W_{e_{\lambda}} \ell_{0} W H_{\phi_{e}},
$$

due to Proposition 3.1 and also taking into account that, because $e_{\lambda} \in H_{-}^{\infty}(\mathbb{R})$, $W H_{e_{\lambda}}=W_{e_{\lambda}}$. Since $\phi_{-} \in \mathcal{G} A P^{-}$, by the characterization of $\mathcal{G} A P^{-}$, there exists a $\psi \in A P^{-}$such that $\phi_{-}=e^{\psi}$. Thus, the mean motion of $\phi_{-}$is zero and due to the Gohberg-Feldman/Coburn-Douglas Theorem (stated in the first section), $W_{\phi_{-}}$ is invertible. From Proposition 3.2] we know that $W H_{\phi_{e}}$ is invertible. Therefore, since $\ell_{0}: L^{2}\left(\mathbb{R}_{+}\right) \rightarrow L_{+}^{2}(\mathbb{R})$ is also an invertible operator, (5.2) shows that $W H_{\phi}$ is equivalent to $W_{e_{\lambda}}$. Once again, by the Theorem of Gohberg-Feldman/CoburnDouglas, since the mean motion of $e_{\lambda}$ is $\lambda<0$, we have that the operator $W_{e_{\lambda}}$ is properly $d$-normal and right-invertible. Consequently, due to the equivalence relation (5.2), the operator $W H_{\phi}$ is also properly $d$-normal and right-invertible. This completes the proof of part (a).

Part (b) can be derived from part (a) by passage to adjoint operators.

Finally, let us now suppose that $\lambda=0$. Then $\phi=\phi_{-} \phi_{e}$ and $W H_{\phi}=$ $W_{\phi_{-}} \ell_{0} W H_{\phi_{e}}$. Since $W_{\phi_{-}}$and $W H_{\phi_{e}}$ are invertible, then $W H_{\phi}$ is also invertible.

THEOREM 5.2. If $\phi \in \mathcal{G} A P$ admit an AP asymmetric factorization

$$
\phi=\phi_{-} e_{\lambda} \phi_{e},
$$

then we obtain a reflexive generalized inverse of $W_{\phi}$ defined by

$$
W H_{\phi}^{-}=\ell_{0} r_{+} \mathcal{F}^{-1} \phi_{e}^{-1} \cdot \mathcal{F} \ell^{e} r_{+} \mathcal{F}^{-1} e_{-\lambda} \cdot \mathcal{F} \ell^{e} r_{+} \mathcal{F}^{-1} \phi_{-}^{-1} \cdot \mathcal{F} \ell: L^{2}\left(\mathbb{R}_{+}\right) \rightarrow L_{+}^{2}(\mathbb{R}),
$$

where $\ell: L^{2}\left(\mathbb{R}_{+}\right) \rightarrow L^{2}(\mathbb{R})$ denotes an arbitrary extension operator.

Additionally, in a more detailed way:

(a) if $\lambda<0$, then $W_{\phi}^{-}$is the right-inverse of $W H_{\phi}$;

(b) if $\lambda>0$, then $W_{\phi}^{-}$is the left-inverse of $W_{\phi}$;

(c) if $\lambda=0$, then $W_{\phi}^{-}$is the inverse of $W H_{\phi}$.

Proof. From the $A P$ asymmetric factorization $\phi=\phi_{-} e_{\lambda} \phi_{e}$, it directly follows that

$$
W H_{\phi}=r_{+} A_{-} E A_{e} \ell^{e} r_{+},
$$

where $A_{-}=\mathcal{F}^{-1} \phi_{-} \cdot \mathcal{F}, E=\mathcal{F}^{-1} e_{\lambda} \cdot \mathcal{F}$ and $A_{e}=\mathcal{F}^{-1} \phi_{e} \cdot \mathcal{F}$.

(i) If $\lambda \leq 0$, consider

$$
\begin{aligned}
W H_{\phi} W H_{\phi}^{-} & =r_{+} A_{-} E A_{e} \ell^{e} r_{+} \ell_{0} r_{+} A_{e}^{-1} \ell^{e} r_{+} E^{-1} \ell^{e} r_{+} A_{-}^{-1} \ell \\
& =r_{+} A_{-} E A_{e} \ell^{e} r_{+} A_{e}^{-1} \ell^{e} r_{+} E^{-1} \ell^{e} r_{+} A_{-}^{-1} \ell,
\end{aligned}
$$

where the term $\ell_{0} r_{+}$was omitted due to the fact that $r_{+} \ell_{0} r_{+}=r_{+}$. Since $A_{e}^{-1}$ preserves the even property of its symbol, we may also drop the first $\ell^{e} r_{+}$term in (5.3), and obtain

$$
W H_{\phi} W H_{\phi}^{-}=r_{+} A_{-} E \ell^{e} r_{+} E^{-1} \ell^{e} r_{+} A_{-}^{-1} \ell .
$$

Additionally, since in the present case (due to $\lambda \leq 0) E^{-1}$ is a plus type factor 9, 24, we have $\ell^{e} r_{+} E^{-1} \ell^{e} r_{+}=E^{-1} \ell^{e} r_{+}$; also because $A_{-}$is a minus type factor it follows

$$
W H_{\phi} W H_{\phi}^{-}=r_{+} A_{-} \ell^{e} r_{+} A_{-}^{-1} \ell=r_{+} \ell=I_{L^{2}\left(\mathbb{R}_{+}\right)},
$$


and we can directly realize that such identities do not depend on the particular choice of the extension operator $\ell$.

(ii) If $\lambda \geq 0$, we will now analyze the composition

$$
W H_{\phi}^{-} W H_{\phi}=\ell_{0} r_{+} A_{e}^{-1} \ell^{e} r_{+} E^{-1} \ell^{e} r_{+} A_{-}^{-1} \ell r_{+} A_{-} E A_{e} \ell^{e} r_{+} .
$$

In the present case $E^{-1}$ is a minus type factor and for this reason $\ell^{e} r_{+} E^{-1} \ell^{e} r_{+}$ $=\ell^{e} r_{+} E^{-1}$. The same reasoning applies to the factor $A_{-}^{-1}$, and therefore the equality (5.6) takes the form

$$
W H_{\phi}^{-} W H_{\phi}=\ell_{0} r_{+} A_{e}^{-1} \ell^{e} r_{+} A_{e} \ell^{e} r_{+}=\ell_{0} r_{+} \ell^{e} r_{+}=\ell_{0} r_{+}=I_{L_{+}^{2}(\mathbb{R})},
$$

where we have used the identity $\ell^{e} r_{+} A_{e} \ell^{e} r_{+}=A_{e} \ell^{e} r_{+}$.

(iii) Intersecting the last two cases, (i) and (ii), it follows that for $\lambda=0$, the operator $W H_{\phi}^{-}$is the (both-sided) inverse of $W H_{\phi}$ (cf. (5.5) and (5.7)).

\section{6. $A P$ Antisymmetric Factorization and its Operator Invertibility Consequences}

Let us recall that $\phi$ is said to admit a right $A P$ factorization $\left[3\right.$ if $\phi=\varphi_{-} e_{\lambda} \varphi_{+}$, where $\varphi_{-} \in \mathcal{G} A P^{-}, \varphi_{+} \in \mathcal{G} A P^{+}$, and $\lambda \in \mathbb{R}$. In addition, if $\lambda=0$ this factorization is called a canonical right AP factorization.

In the present section the $A P$ asymmetric factorization is related to a special case of right AP factorization, which we will call $A P$ antisymmetric factorization. In this new kind of factorization a strong dependence between the left and the right factor occurs.

Definition 6.1. A function $\phi \in \mathcal{G} A P$ admits an $A P$ antisymmetric factorization if it is possible to write

$$
\phi=\phi_{-} e_{2 \lambda} \widetilde{\phi_{-}^{-1}}
$$

where $\lambda \in \mathbb{R}, e_{2 \lambda}(x)=e^{2 i \lambda x}, x \in \mathbb{R}$, and $\phi_{-} \in \mathcal{G} A P^{-}$.

Proposition 6.2. Let $\phi \in \mathcal{G} A P$ and put $\Phi=\phi \widetilde{\phi^{-1}}$.

(a) If $\phi$ admits an $A P$ asymmetric factorization, $\phi=\phi_{-} e_{\lambda} \phi_{e}$, then $\Phi$ admits an $A P$ antisymmetric factorization with the same factor $\phi_{-}$and the same index $\lambda$.

(b) If $\Phi$ admits an $A P$ antisymmetric factorization, $\Phi=\psi_{-} e_{2 \lambda} \widetilde{\psi_{-}^{-1}}$, then $\phi$ admits an $A P$ asymmetric factorization with the same minus factor $\psi_{-}$, the same index $\lambda$ and the even factor $\phi_{e}=e_{-\lambda} \psi_{-}^{-1} \phi$.

Proof. (a) From the $A P$ asymmetric factorization of $\phi, \phi=\phi_{-} e_{\lambda} \phi_{e}$, we have

$$
\widetilde{\phi^{-1}}=\phi_{e}^{-1} e_{\lambda} \widetilde{\phi_{-}^{-1}}
$$

with $\phi_{-} \in \mathcal{G} A P^{-}$. Hence

$$
\Phi=\phi \widetilde{\phi^{-1}}=\phi_{-} e_{2 \lambda} \widetilde{\phi_{-}^{-1}} .
$$

(b) It follows from the definition of the factor $\phi_{e}$ that $\phi=\psi_{-} e_{\lambda} \phi_{e}$. Thus it remains to prove that $\phi_{e}$ is an even function. Once again by the definition of $\phi_{e}$, we obtain

$$
\widetilde{\phi_{e}}=e_{\lambda} \widetilde{\psi_{-}^{-1}} \widetilde{\phi}=e_{\lambda} e_{-2 \lambda} \psi_{-}^{-1} \phi=e_{-\lambda} \psi_{-}^{-1} \phi=\phi_{e},
$$

since $\widetilde{\psi_{-}^{-1}} \widetilde{\phi}=e_{-2 \lambda} \psi_{-}^{-1} \phi$ (due to the $A P$ antisymmetric factorization of $\Phi$ ). Therefore $\phi_{e}$ is an even function. 
Theorem 6.3. Let $\phi \in \mathcal{G} A P$. If $\phi$ admits a right AP factorization,

$$
\phi=\varphi_{-} e_{\lambda} \varphi_{+},
$$

then $\phi$ admits an AP asymmetric factorization,

$$
\phi=\phi_{-} e_{\lambda} \phi_{e}
$$

with $\phi_{-}=\varphi_{-} \widetilde{\varphi_{+}^{-1}}$, and $\phi_{e}=\widetilde{\varphi_{+}} \varphi_{+}$.

Proof. Suppose that $\phi$ admits a right $A P$ factorization, i.e, $\phi=\varphi_{-} e_{\lambda} \varphi_{+}$, where $\varphi_{-} \in \mathcal{G} A P^{-}, \varphi_{+} \in \mathcal{G} A P^{+}$. Considering $\Phi=\phi \widetilde{\phi^{-1}}$, we have

$$
\Phi=\varphi_{-} e_{\lambda} \varphi_{+} \widetilde{\varphi_{+}^{-1}} e_{\lambda} \widetilde{\varphi_{-}^{-1}}=\varphi_{-} \widetilde{\varphi_{+}^{-1}} e_{2 \lambda} \varphi_{+} \widetilde{\varphi_{-}^{-1}} .
$$

Since $\varphi_{-} \in \mathcal{G} A P^{-}$and $\varphi_{+} \in \mathcal{G} A P^{+}$, then $\widetilde{\varphi_{+}^{-1}} \in \mathcal{G} A P^{-}, \widetilde{\varphi_{-}^{-1}} \in \mathcal{G} A P^{+}$and therefore $\varphi_{-} \widetilde{\varphi_{+}^{-1}} \in \mathcal{G} A P^{-}$and $\varphi_{+} \widetilde{\varphi_{-}^{-1}} \in \mathcal{G} A P^{+}$. Putting $\phi_{-}=\varphi_{-} \widetilde{\varphi_{+}^{-1}}$, it follows from (6.1) that

$$
\Phi=\phi_{-} e_{2 \lambda} \widetilde{\phi_{-}^{-1}} .
$$

Since $\phi_{-} \in \mathcal{G} A P^{-}$, it results that $\Phi$ admits a $A P$ antisymmetric factorization. By Proposition 6.2 that implies that $\phi$ admits a $A P$ asymmetric factorization, $\phi=\phi_{-} e_{\lambda} \phi_{e}$, with $\phi_{e}=e_{-\lambda} \phi_{-}^{-1} \phi$. Rewriting $\phi_{-}$and $\phi_{e}$ by using the factors of the right $A P$ factorization, $\varphi_{-}$and $\varphi_{+}$, we have

$$
\phi_{-}=\varphi_{-} \widetilde{\varphi_{+}^{-1}}, \quad \phi_{e}=\widetilde{\varphi_{+}} \varphi_{+} .
$$

Corollary 6.4. Let $\phi \in \mathcal{G} A P$. If $\phi$ admits a canonical right $A P$ factorization,

$$
\phi=\varphi_{-} \varphi_{+},
$$

then $\phi$ admits a canonical $A P$ asymmetric factorization,

$$
\phi=\phi_{-} \phi_{e},
$$

with $\phi_{-}=\varphi_{-} \widetilde{\varphi_{+}^{-1}}$, and $\phi_{e}=\widetilde{\varphi_{+}} \varphi_{+}$.

Proof. The result is a direct consequence of Theorem 6.3 if we take there $\lambda=0$.

TheOREM 6.5. Let $\phi \in \mathcal{G} A P$. If $W_{\phi}$ is invertible with $\phi$ having a canonical right AP factorization, then $W H_{\phi}$ is invertible.

Proof. Suppose that $\phi=\varphi_{-} \varphi_{+}$is a canonical right $A P$ factorization of $\phi$. By Corollary 6.4 $\phi$ admits a canonical $A P$ asymmetric factorization, $\phi=\phi_{-} \phi_{e}$, where

$$
\phi_{-}=\varphi_{-} \widetilde{\varphi_{+}^{-1}}, \quad \phi_{e}=\widetilde{\varphi_{+}} \varphi_{+} .
$$

From Theorem 5.1 it follows that $W H_{\phi}$ is an invertible operator.

Acknowledgements: The work was supported by Fundação para a Ciência e a Tecnologia through Unidade de Investigação Matemática e Aplicações of University of Aveiro, Portugal. 


\section{References}

[1] E.L. Basor and T. Ehrhardt, Factorization theory for a class of Toeplitz + Hankel operators, J. Oper. Theory, 51 (2004), 411-433.

[2] E.L. Basor, T. Ehrhardt and H. Widom, On the determinant of a certain Wiener-Hopf + Hankel operator, Integral Equations Operator Theory, 47 (2003), 275-288.

[3] A. Böttcher, Yu.I. Karlovich and I.M. Spitkovsky, Convolution Operators and Factorization of Almost Periodic Matrix Functions, Birkhäuser, Basel (2002).

[4] A. Böttcher, B. Silbermann, Analysis of Toeplitz Operators, Akademie- Verlag, Berlin (1989), and Springer-Verlag, Berlin (1990).

[5] L.P. Castro, Regularity of convolution type operators with PC symbols in Bessel potential spaces over two finite intervals, Math. Nachr., 261/262 (2003), 23-36.

[6] L.P. Castro and F.-O. Speck, Regularity properties and generelized inverses of delta-related operators, Z. Anal. Anwend., 17 (1998), 577-598.

[7] L.P. Castro, F.-O. Speck and F.S. Teixeira, Explicit solution of a Dirichlet-Neumann wedge diffraction problem with a strip, J. Integral Equations Appl., 15 (2003), 359-383.

[8] L.P. Castro, F.-O. Speck and F.S. Teixeira, On a class of wedge diffraction problems posted by Erhard Meister, Oper. Theory Adv. Appl., 147 (2004), 211-238.

[9] L.P. Castro, F.-O. Speck and F.S. Teixeira, A direct approach to convolution type operators with symmetry, Math. Nachr., 269-270 (2004), 73-85.

[10] L.A. Coburn and R.G. Douglas, Translation operators on the half-line, Proc. Natl. Acad. Sci. USA, 62 (1969), 1010-1013.

[11] T. Ehrhardt, Invertibility theory for Toeplitz plus Hankel operators and singular integral operators with flip, J. Funct. Anal., 208 (2004), 64-106.

[12] I. Gohberg and I.A. Fel'dman, On Wiener-Hopf integral-difference equations, Sov. Math., Dokl., 9 (1968), 1312-1316.

[13] H. Hankel, Ueber eine besondere Classe der symmetrishchen Determinanten, (Leipziger) Dissertation, Göttingen (1861).

[14] L. Kronecker, Zur Theorie der Elimination einer Variabeln aus zwei algebraischen Gleichungen, Monatsber. Königl. Preuss. Akad. Wiss., Berlin (1881), 535-600.

[15] A.B. Lebre, E. Meister, F.S. Teixeira, Some results on the invertibility of Wiener-Hopf-Hankel operators, Z. Anal. Anwendungen, 11 (1992), 57-76.

[16] E. Meister, F.-O. Speck, F.S. Teixeira, Wiener-Hopf-Hankel operators for some wedge diffraction problems with mixed boundary conditions, J. Integral Equations Appl., 4 (1992), 229255.

[17] Z. Nehari, On bounded bilinear forms, Ann. Math., 65 (1957), 153-162.

[18] A.P. Nolasco and L.P. Castro, Factorization of Wiener-Hopf plus Hankel operators with APW Fourier symbols, Int. J. Pure Appl. Math., 14 (2004), 537-550.

[19] S.C. Power, $C^{*}$-algebras generated by Hankel operators and Toeplitz operators, J. Funct. Anal., 31 (1979), 52-68.

[20] S.C. Power, Hankel operators with PQC symbols and singular integral operators, Proc. London Math. Soc., 41 (1980), 45-65.

[21] S.C. Power, Hankel Operators on Hilbert Space, Pitman (Advanced Publishing Program), Boston (1982).

[22] S. Roch and B. Silbermann, Algebras of Convolution Operators and their Image in the Calkin Algebra, Akademie der Wissenschaften der DDR, Karl-Weierstrass-Institut für Mathematik, Berlin (1990).

[23] B. Silbermann, The $C^{*}$-algebra generated by Toeplitz and Hankel operators with piecewise quasicontinuous symbols, Integral Equations Operator Theory, 10 (1987), 730-738.

[24] F.-O. Speck, General Wiener-Hopf Factorization Methods, Pitman, London (1985).

[25] F.S. Teixeira, Diffraction by a rectangular wedge: Wiener-Hopf-Hankel formulation, Integral Equations Operator Theory, 14 (1991), 436-454.

[26] O. Toeplitz, Zur Theorie der quadratischen und bilinearen Formen von unendlichvielen Veränderlichen, Math. Annalen, 70 (1911), 351-376.

[27] Wiener, N. and Hopf, E., Über eine Klasse singulärer Integralgleichungen, Sitzungsber. Preuss. Akad. Wiss., Phys.-Math. Kl., 30/32 (1931), 696-706. 
Department of Mathematics, University of Aveiro, 3810-193 Aveiro, Portugal

E-mail address: anolasco@mat.ua.pt

Department of Mathematics, University of Aveiro, 3810-193 Aveiro, Portugal

E-mail address: lcastro@mat.ua.pt

$U R L:$ http://www.mat.ua.pt/lcastro/ 